\title{
Erfahrungen aus dem Peer-Review-Verfahren
}

Das in den HELIOS-Kliniken entwickelte und etablierte Peer-Review-Verfahren dient der Qualitätskontrolle auf dem Boden von Routinedaten. Unter diesen ist die Krankenhausletalität eine über Entlassdaten vollständig erfassbare und deshalb nahezu nicht beeinflussbare Kenngröße, die für jede gewünschte Patientengruppe definiert werden kann. Sie dient insbesondere als Marker für Qualitätskontrollen nach chirurgischen Eingriffen.

Ziel des Peer Review-Verfahrens ist es, deutlich vom vereinbarten Ziel abweichende Kliniken einer Ursachenanalyse zu unterziehen.

\section{Peer-Review | Analysekriterien \\ $\checkmark$}

Die Bewertung und Einstufung des gesamten Behandlungsprozesses erfolgt nach folgenden Kriterien des HELIOS Peer Review-Verfahrens:

- Waren Diagnostik und Behandlung adäquat und zeitgerecht sowie der Behandlungsprozess zielführend und zeitnah kritisch hinterfragt?

- Sind Indikation zur Operation, Intervention und Intensivtherapie angemessen und rechtzeitig erfolgt?

- Wurden Behandlungsleitlinien und Standards berücksichtigt?

- War die Dokumentation umfassend und schlüssig?

- Lag eine reibungslose interdisziplinäre Zusammenarbeit vor?

- Erfolgten Kontrollen der Behandlungsverläufe?

Finden sich mehrere Mängel bei der Einzelanalyse des gesamten Behandlungsprozesses, so resultiert dies in einem vermuteten oder sicheren Optimierungspotential.

Neben dem abteilungsbezogenen wurde seit zwei Jahren auch ein zentrales Peer-Review-Verfahren eingeführt, welches die Todesfälle der sogenannten Low-Risk-Operationen überprüft. Hier handelt es sich um Eingriffe wie die Entfernung einer Gallenblase oder die Operation einer Leistenhernie, bei denen eine Sterblichkeit von deutlich unter $1 \%$ erwartet wird.

\section{Ergebnisse \\ $\checkmark$}

Ein Optimierungspotential von 30\% ist generell zu erwarten. Während beim abteilungsspezifischen Review individuell wiederkehrende Pro- blemkreise in der OP-Indikation, der interdisziplinären Zusammenarbeit, der operativen Ausführung oder der postoperativen Überwachung und Kontrolle inklusive intensivmedizinischer Betreuung identifiziert werden können, finden sich beim Zentralen Review in der Analyse aller im Low-Risk-Bereich verstorbenen Patienten in allen vorgenannten Bereichen ungefähr gleich häufig vertretene Optimierungspotentiale, die hier insgesamt bei $70 \%$ lagen.

\section{Zusammenfassung}

Insbesondere die Analyse der Low-Risk-Fälle präsentiert das gesamte Repertoire der Fehlermöglichkeiten von Indikationsstellung über operative Ausführung bis hin zum perioperativen Management. Die Erkennung von Risiko-Patienten, eine differenzierte, risikoadaptierte und zeitgerechte OP-Indikation sowie eine optimierte postoperative Überwachung mit rechtzeitigem Erkennen von Problemsituationen nebst einer leitliniengerechten Intensivtherapie und einer reibungslosen interdisziplinären Zusammenarbeit stehen im Fokus der Betrachtung.

Autorenerklärung: Es bestehen keine finanziellen Interessenkonflikte in Zusammenhang mit diesem Artikel.

\section{H. Zirngibl}

Qualitätsmanagement

Schlüsselwörter

Prozessanalyse

Optimierungspotenzial

perioperatives Management

Key words

analysis of processes

potential for improvement

perioperative management

Institut

Chirurgische Klinik, HELIOS Klinikum Wuppertal

Bibliografie

Dol 10.1055/s-0028-1085591 Dtsch Med Wochenschr 2008; 133: S 147 . (c) Georg Thieme Verlag KG Stuttgart · New York . ISSN 0012-0472

Korrespondenz Prof. Dr. Hubert Zirngibl Direktor chirurgische Klinik HELIOS Klinikum Wuppertal Heusnerstr. 40 42283 Wuppertal Tel. +49 (202) 8962320 eMail hubert.zirngibl@ helios-kliniken.de 